\title{
Pharmaceutical patent landscape for cancer immunotherapy: an interview with Gilles Berger
}

\author{
Gilles Berger*,1,2(D) \\ ${ }^{1}$ Harvey Cushing Neuro-Oncology Laboratories, Department of Neurosurgery, Brigham \& Women's Hospital, Harvard Medical \\ School, Boston, MA 02115, USA \\ ${ }^{2}$ Microbiology, Bioorganic \& Macromolecular Chemistry, Faculty of Pharmacy, Université libre de Bruxelles, Boulevard du Triomphe, \\ 1050 Brussels, Belgium \\ *Author for correspondence: gpberger@bwh.harvard.edu
}

"Preclinical studies have shown powerful and promising responses for intratumoral treatment with STING agonists in an array of tumor types"

Dr Gilles Berger is a chemist and pharmacist with broad interest in organic and theoretical chemistry, drug discovery and design, biomedical sciences and oncology. He has worked as a Research Fellow in Brussels, Paris, Montreal and at MIT in Boston, where he has gathered hands-on experience at the interface of drug design, nanotechnologies, biology and human disease. He has been involved in project management and mentoring and has in depth experience in collaborative and multi-disciplinary projects, with a proven track record of publications in various fields, in collaboration with research groups from all around Europe, Canada and the US. In recent years, he has developed as a translational scientist, allowing the use of his complementary multi-disciplinary skills toward the advancement of fundamental projects, such as organocatalysis, theoretical chemistry or halogen bonding; as well as in applications like drug discovery, as evidenced by his numerous publications aimed at developing novel anticancer medicines and other agents. He is currently a Research Fellow of the Harvard Medical School, a Research Associate at MIT and a Fellow of the Belgian Science Foundation.

First draft submitted: 25 September 2019; Accepted for publication: 25 September 2019; Published online: 15 October 2019

Can you give us a short summary of your recent article 'Current patent \& clinical status of stimulator of interferon genes (STING) agonists for cancer immunotherapy'?

We wanted to give a concise overview on the current stimulator of interferon genes (STING) patents and trials, considering how fast the field has been developing and the excitement it has created in the past few years. Cancer immunotherapy itself has brought new treatment modalities and unprecedented responses in advanced and metastatic stages of the cancer disease. However, the response rate among patients is rather limited (10-40\%), and new therapies or adjuvants are sought to help increase this ratio of responders.

\section{What role do the STING play in mediating cancer?}

Its role has been recently revealed as a critical mediator of innate immunity, both against pathogens and cancer. The pathway is triggered by cGAS, which is a sensor for cytosolic DNA, and whose presence inside cells either indicates an infection (in the case of foreign DNA) or nearby tumor cells, from which tumor DNA is released. cGAS can bind to double-stranded DNA and will in turn catalyze the synthesis of a cyclic dinucleotide, cGAMP, which will trigger the pathway through binding to STING. This ultimately leads to a strong immune response via the production of type I interferons and other cytokines. 


\section{What are the advantages of targeting the cGMP-AMP synthase (cGAS)-STING-IRF3 pathway in cancer immunotherapy?}

Activation of the innate immune system and the interferon response promote tumor inflammation and facilitates antitumor $\mathrm{T}$ cell responses, notably by the recruitment, activation and expansion of $\mathrm{CD} 8^{+} \mathrm{T}$ cells. This subpopulation of $\mathrm{T}$ cells and, more generally, the presence of tumor infiltrating lymphocytes within the tumor microenvironment have been shown as a crucial parameter for cancer prognosis and clinical outcome, being more predictive than the classical tumor-node-metastasis staging. It is then extremely appealing to 'heat up' the tumor and attract immune infiltrates by pharmacological activation of the cGAS-STING-IRF3 pathway. Preclinical studies have shown powerful and promising responses for intratumoral treatment with STING agonists in an array of tumor types.

\section{Are there any current highlights for therapeutic patents targeting this pathway?}

The most advanced compound on the way to clinics and patients is the Aduro Biotech's candidate, ADU-S100. It is an interestingly designed cyclic dinucleotide analog, in which nonbridging oxygens from the phosphodiester linkages have been replaced by sulfur atoms to make phosphorothioates that are resistant to enzymatic cleavage. It has shown exciting results in preclinical models and is currently being tested in multiple clinical trials in cutaneously accessible tumors, including head-and-neck cancer, melanoma and multiple other cancers. Preliminary clinical data in ongoing early phase trials is encouraging but it is still too early to draw firm conclusions.

\section{Comparing nucleotidic STING agonists \& non-nucleotidic STING agonists, which pharmaceutical patent landscape currently has more promise?}

The development of non-nucleotidic agonists has been lagging a little but, from a medicinal chemist view, they represent a more viable alternative, considering the major drawbacks cyclic dinucleotides are facing: instability of the phosphodiester linkages, a negative net charge that hampers membrane diffusion and cell penetration and a complex chemical synthesis. Non-nucleotidic analogs would certainly bring a better pharmacokinetic profile and simplify things for the use of STING agonists in cancer patients.

\section{How would you like to see this field develop in the future?}

I sincerely wish, and would like to see, these treatments hit the clinics and cure cancer patients! I mostly see it as an adjuvant to other immunotherapy regimens, especially to immune checkpoint blockade. The Achilles' heel of these treatments is precisely that 'cold', non-immunogenic tumors fail to respond, and generating tumor inflammation, by activation of the STING pathway and the interferon response, could help trigger anticancer immunity in non-responding patients, leading to long-lasting responses and remissions.

\section{Disclaimer}

The opinions expressed in this interview are those of the interviewee and do not necessarily reflect the views of Future Science Ltd.

\section{Financial \& competing interests disclosure}

The author has no relevant affiliations or financial involvement with any organization or entity with a financial interest in or financial conflict with the subject matter or materials discussed in the manuscript. This includes employment, consultancies, honoraria, stock ownership or options, expert testimony, grants or patents received or pending, or royalties.

No writing assistance was utilized in the production of this manuscript. 